\title{
Bioefficacy of Various Fungicides against Rhizoctonia bataticola, Causing Dry Root Rot of Soybean
}

\author{
R.C. Agale ${ }^{1 *}$, A.P.Suryawanshi ${ }^{2}$, R.R. Rathod $^{2}$ and K.T. Apet ${ }^{1}$ \\ ${ }^{1}$ Department of Plant Pathology, Vasantrao Naik Marathwada Krishi Vidyapeeth, \\ Parbhani- 431 402, India \\ ${ }^{2}$ Department of Plant Pathology, Dr. Balasaheb Sawant Konkan Krishi Vidyapeeth, \\ Dapoli-415 712, India \\ *Corresponding author
}

\begin{tabular}{|c|c|}
\hline & A B S T R A C T \\
\hline $\begin{array}{l}\text { K e y w o r d s } \\
\text { Glycine max, Rhizoctonia } \\
\text { bataticola, Dry root rot, } \\
\text { Inhibition, Fungicides }\end{array}$ & \multirow{3}{*}{$\begin{array}{l}\text { Dry root rot caused by Rhizoctonia bataticola (Taub) Butler, is one of the most widely } \\
\text { distributed and destructive disease of soybean [Glycine max (L.) Merril], causing } \\
\text { accountable quantitative and qualitative losses. All of the } 17 \text { fungicides (seven systemic, } \\
\text { six contact and four combi- fungicides) evaluated in vitro were found effective with } \\
\text { significant mycelial growth inhibition of } R \text {. bataticola, over untreated control. However, } \\
\text { carbendazim } 50 \% \text { WP (@ 500, } 1000 \text { and } 1500 \text { ppm), carboxin } 37.5 \%+\text { thiram } 37.5 \% \text { WP } \\
\text { (@ } 1500,2000 \text { and } 2500 \text { ppm) and carbendazim } 12 \% \text { WP + Mancozeb } 63 \% \text { WP (@ } 2000 \\
\text { and } 2500 \mathrm{ppm} \text { ) resulted with cent per cent (100\%) mycelia growth inhibition, followed by } \\
\text { Thiophanate methyl } 70 \% \text { WP with average mycelia growth inhibition of } 93.57 \text { per cent, } \\
\text { Captan } 50 \% \text { WP }(89.48 \%) \text { and Hexanconazole 5\% EC (87.62\%), over untreated control. }\end{array}$} \\
\hline Article Info & \\
\hline $\begin{array}{l}\text { Accepted: } \\
\text { 15 September } 2018 \\
\text { Available Online: } \\
\text { 10 October } 2018\end{array}$ & \\
\hline
\end{tabular}

\section{Introduction}

Soybean [Glycine max (L.) Merril] is a major commodity traded in world markets and currently, the world's prime oilseed crop (Sonka et al., 2004). Among various factors contributing to yield losses in Soybean, diseases caused by fungal, bacterial, viral and nematode pathogens are the major constraints. During recent past, the soybean crop has badly been affected by the pathogenic fungus Rhizoctonia bataticola (Taub.) Butler, causing dry root rot disease. The disease was previously supposed to be of minor importance in soybean and other crops, but now has been emerging as a major threat; inflicting potential seed yield losses of 3-36\% (Sangeetha and Jahagirdar, 2013) and also reduce plant population per unit area upto 77 per cent (Muthusamy and Mariappan, 1991). In Marathwada region, soybean is being grown on large scale as rainfed crop, due to which the crop is frequently subjected to the attack by dry root rot $(R$. bataticola $)$ and charcoal rot (M. phaseolina) diseases. $R$. bataticola, being basically soil borne, with wider adaptability and longer survivability in soil, render difficult to control it with chemicals alone and the use of chemicals is non-feasible, uneconomical and hazardous to 
the ecosystem. Hence, it is imperative to exploit alternative and eco-friendly disease management practices for such soil borne diseases.

\section{Materials and Methods}

Efficacy of seven systemic (each @ 500, 1000 and $1500 \mathrm{ppm}$ ), six contact and four combifungicides (each @ 1500, 2000 and 2500 ppm) were evaluated in vitro against $R$. bataticola (Rb4 isolate), applying Poisoned food technique (Nene and Thapliyal, 1993) and using Potato dextrose agar (PDA) as basal culture medium. Based on active ingredient, requisite quantity of the test fungicides was separately mixed thoroughly with autoclaved and cooled $\left(45^{\circ} \mathrm{C}\right)$ PDA medium in sterile glass conical flasks (250 ml. capacity) to obtain desired concentrations. This PDA medium amended separately with the test fungicides was poured $(20 \mathrm{ml} /$ plate $)$ aseptically in sterile glass Petri plates $(90 \mathrm{~mm}$ dia.) and allowed to solidify at room temperature.

For each of the test fungicide and its desired concentrations, three plates / treatment / replication were maintained. After solidification of the PDA medium, all the plates were inoculated aseptically by placing in the centre a $5 \mathrm{~mm}$ pure culture disc, obtained from actively growing 7 days old pure culture of $R$. bataticola and incubated in an inverted position at $28 \pm 2{ }^{\circ} \mathrm{C}$. Petri plates filled with plain PDA (without any fungicide) and inoculated with the pure culture disc of $R$. bataticola were maintained as untreated control. Two separate experiments were planned in Completely Randomized Design (CRD) and all the treatments replicated thrice.

Observations on radial mycelial growth / colony diameter were recorded at an interval of $24 \mathrm{hrs}$, continued till untreated control plates were fully covered with mycelial growth of the test pathogen and averaged finally. Per cent inhibition of the test pathogen with the test fungicides, over untreated control was calculated by applying following formula (Vincent, 1927).

Per cent inhibition $=\frac{\text { C }- \text { T }}{\text { C }}$

Where,

$\mathrm{C}=$ growth of the test fungus in untreated control plate

$\mathrm{T}=$ growth of the test fungus in treated plate

\section{Results and Discussion}

\section{In vitro efficacy of systemic fungicides}

\section{Mycelial growth}

Results (Plate I, Table 1 and Fig. 1) revealed that all of the seven systemic fungicides tested exhibited a wide range of radial mycelial growth of $R$. bataticola and it was decreased drastically with increase in their concentrations.

The systemic fungicides resulted with mycelia growth in the range of 00.00 to $26.95 \mathrm{~mm}$, 00.00 to $22.39 \mathrm{~mm}$ and 00.00 to $20.80 \mathrm{~mm}$, respectively@500, 1000 and 1500 ppm, as against $90.00 \mathrm{~mm}$ in untreated control.

Among the systemic fungicides, Carbendazim $50 \%$ WP resulted with none of the mycelial growth at 500, 1000 and $1500 \mathrm{ppm}$. The next fungicides with significantly least mycelial growth were Thiophanate methyl $70 \%$ WP (7.22, 5.08 and $5.06 \mathrm{~mm}$, respectively), followed by Hexaconazole 5\% EC (14.92, 11.02 and $7.49 \mathrm{~mm}$, respectively), Tebuconazole $29.9 \%$ EC (17.85, 16.80 and $14.90 \mathrm{~mm}$, respectively), Difenconazole $25 \%$ 
EC (20.95, 12.26 and $7.37 \mathrm{~mm}$, respectively) Benomyl 50\% WP (21.19, 20.13 and 18.95 $\mathrm{mm}$, respectively) and Propiconazole $25 \%$ EC (26.95, 22.39 and $13.06 \mathrm{~mm}$, respectively), respectively@500,1000 and 1500 ppm.

Average radial mycelial growth of the test pathogen ranged from $00.00 \mathrm{~mm}$ (Carbendazim) to $20.80 \mathrm{~mm}$ (Propiconazole). However, there was none of the average mycelial growth with Carbendazim.

The fungicides with next lowest average mycelial growth were Thiophanate methyl (5.79 $\mathrm{mm})$, followed by Hexaconazole (11.14 $\mathrm{mm})$ Difenconazole (13.43 $\mathrm{mm})$, Tebuconazole (16.52 mm), Benomyl (20.09 $\mathrm{mm}$ ) and Propiconazole (20.80).

\section{Mycelial growth inhibition}

Results (Table 1, Plate I and Fig. 1) revealed that all of the systemic fungicides tested (each @ 500, 1000 and 1500 ppm) significantly inhibited mycelial growth of $R$. bataticola, over untreated control and it was found to increased with increase in concentrations of the fungicides tested.

The mycelia growth inhibition resulted with the test systemic fungicides ranged from 70.00 to $100.00,75.12$ to 100.00 and 78.94 to 100.00 per cent, respectively @ 500, 1000 and 1500 ppm, over untreated control.

However, Carbendazim 50\% WP (@ 500, 1000 and $1500 \mathrm{ppm}$ ) resulted with cent per cent (100\%) mycelial growth inhibition, followed by Thiophanate methyl $70 \%$ WP (91.97, 94.36 and $94.38 \%$, respectively), Tebuconazole 29.9\% EC (90.35, 81.34 and $83.45 \%$, respectively), Hexaconazole $5 \%$ EC (83.42, 87.76 and $91.68 \%$, respectively), Difenconazole 25\% EC (77.03, 86.38 and $91.81 \%$, respectively), Benomyl 50\% WP (76.45, 77.64 and $78.94 \%$, respectively) and
Propiconazole 25\% EC (70.06, 75.12 and $85.49 \%$, respectively), respectively @ 500, 1000 and $1500 \mathrm{ppm}$.

Average mycelial growth inhibition recorded with the test systemic fungicides ranged from 76.89 (Propiconazole) to 100 (Carbendazim) per cent. However, it was cent per cent with Carbendazim (100\%), followed by Thiophanate methyl (94.57\%), Hexaconazole (87.62\%), Difenconazole (85.07\%), Tebuconazole (85.05\%), Benomyl (77.68\%) and Propiconazole (76.89\%). Thus, all of the systemic fungicides tested were found fungistatic against $R$. bataticola and significantly inhibited its mycelial growth, over untreated control. However, the systemic fungicides found most effective in the order of merit were Carbendazim 50\% WP > Thiophanate methyl 70\% WP > Hexaconazole $5 \%$ EC > Difenconazole 25\% EC > Tebuconazole 29.9\% EC > Benomyl 50\% WP $>$ Propiconazole $25 \%$ EC.

\section{In vitro efficacy of contact and combi- fungicides}

\section{Mycelial growth}

Results (Table 2, Plate II and Fig. 2) revealed that all of the six contact and four combifungicides tested exhibited a wide range of radial mycelial growth of $R$. bataticola and was decreased drastically with increase in concentrations of the test fungicides from 1500 to $2500 \mathrm{ppm}$.

The test fungicides resulted with mycelia growth in the range of 00.00 to $55.62 \mathrm{~mm}$, 00.00 to $48.94 \mathrm{~mm}$ and 00.00 to $43.75 \mathrm{~mm}$, respectively @ 1500, 2000 and 2500 ppm. However, the combi-fungicides Carboxin $37.5 \%$ + Thiram $37.5 \% \mathrm{WP}$ at all three concentrations and Carbendazim $12 \%+$ Mancozeb 63 \% WP @ 2000 and 2500 ppm resulted with none of the mycelial growth. 
Table.1 In vitro efficacy of systemic fungicides against $R$. bataticola

\begin{tabular}{|c|c|c|c|c|c|c|c|c|c|}
\hline \multirow{2}{*}{$\begin{array}{l}\text { Tr. } \\
\text { No. }\end{array}$} & \multirow[t]{2}{*}{ Treatments } & \multicolumn{3}{|c|}{ Colony Dia. *(mm) at ppm } & \multirow{2}{*}{$\begin{array}{l}\text { Av. } \\
(\mathbf{m m})\end{array}$} & \multicolumn{3}{|c|}{$\%$ Inhibition* at ppm } & \multirow{2}{*}{$\begin{array}{c}\text { Av. inhibition } \\
(\%)\end{array}$} \\
\hline & & 500 & 1000 & 1500 & & 500 & 1000 & 1500 & \\
\hline$\overline{T_{1}}$ & $\begin{array}{l}\text { Carbendazim } 50 \% \\
\text { WP }\end{array}$ & 0.00 & 0.00 & 0.00 & 0.00 & $\begin{array}{l}100.00 \\
(90.00)\end{array}$ & $\begin{array}{l}100.00 \\
(90.00)\end{array}$ & $\begin{array}{l}100.00 \\
(90.00)\end{array}$ & $\begin{array}{l}100.00 \\
(90.00)\end{array}$ \\
\hline $\mathrm{T}_{2}$ & $\begin{array}{l}\text { Thiophanate methyl } \\
70 \% \text { WP }\end{array}$ & 7.22 & 5.08 & 5.06 & 5.79 & $\begin{array}{l}91.97 \\
(73.54)\end{array}$ & $\begin{array}{c}94.36 \\
(76.26)\end{array}$ & $\begin{array}{c}94.38 \\
(76.29)\end{array}$ & $\begin{array}{c}93.57 \\
(75.31)\end{array}$ \\
\hline$\overline{T_{3}}$ & Benomyl 50\% WP & 21.19 & 20.13 & 18.95 & 20.09 & $\begin{array}{l}76.45 \\
(60.97)\end{array}$ & $\begin{array}{c}77.64 \\
(61.78)\end{array}$ & $\begin{array}{c}78.94 \\
(62.68)\end{array}$ & $\begin{array}{l}77.68 \\
(61.80)\end{array}$ \\
\hline $\mathbf{T}_{4}$ & $\begin{array}{l}\text { Difenconazole } 25 \% \\
\text { EC }\end{array}$ & 20.67 & 12.26 & 7.37 & 13.43 & $\begin{array}{l}77.03 \\
(61.36)\end{array}$ & $\begin{array}{c}86.38 \\
(68.34)\end{array}$ & $\begin{array}{c}91.81 \\
(73.37)\end{array}$ & $\begin{array}{c}85.07 \\
(67.27)\end{array}$ \\
\hline$\overline{T_{5}}$ & $\begin{array}{l}\text { Propiconazole } 25 \% \\
\text { EC }\end{array}$ & 26.95 & 22.39 & 13.06 & 20.80 & $\begin{array}{c}70.06 \\
(56.83)\end{array}$ & $\begin{array}{l}75.12 \\
(60.08)\end{array}$ & $\begin{array}{c}85.49 \\
(67.61)\end{array}$ & $\begin{array}{c}76.89 \\
(61.27)\end{array}$ \\
\hline$T_{6}$ & $\begin{array}{l}\text { Tebuconazole } 29.9 \% \\
\text { EC }\end{array}$ & 17.85 & 16.80 & 14.90 & 16.52 & $\begin{array}{l}90.35 \\
(71.90)\end{array}$ & $\begin{array}{c}81.34 \\
(64.41)\end{array}$ & $\begin{array}{r}83.45 \\
(65.99)\end{array}$ & $\begin{array}{c}85.05 \\
(67.25)\end{array}$ \\
\hline $\mathbf{T}_{7}$ & $\begin{array}{l}\text { Hexaconazole } 5 \% \\
\text { EC }\end{array}$ & 14.92 & 11.02 & 7.49 & 11.14 & $\begin{array}{c}83.42 \\
(65.97)\end{array}$ & $\begin{array}{c}87.76 \\
(69.52)\end{array}$ & $\begin{array}{c}91.68 \\
(73.23)\end{array}$ & $\begin{array}{c}87.62 \\
(69.40)\end{array}$ \\
\hline \multirow[t]{6}{*}{$\overline{T_{8}}$} & Control (untreated) & 90.00 & 90.00 & 90.00 & 90.00 & $\begin{array}{c}0.00 \\
(0.00)\end{array}$ & $\begin{array}{c}0.00 \\
(0.00)\end{array}$ & $\begin{array}{c}0.00 \\
(0.00)\end{array}$ & $\begin{array}{c}0.00 \\
(0.00)\end{array}$ \\
\hline & S.E. \pm & 0.42 & 0.20 & 0.15 & 0.26 & 0.31 & 0.18 & 0.15 & 0.21 \\
\hline & C.D. $(\mathbf{P}=0.01)$ & 1.29 & 0.60 & 0.44 & 0.78 & 0.92 & 0.53 & 0.44 & 0.63 \\
\hline & & & & & & Fungicides & Conc. & F X C & - \\
\hline & S.E. \pm & & & & & 2.37 & 0.10 & 0.30 & - \\
\hline & C.D. $(P=0.01)$ & & & & & 7.19 & 0.30 & 0.84 & - \\
\hline
\end{tabular}

*: Mean of three replications, Dia: Diameter, Av.: Average

Figures in parentheses are arcsine transformed values

Table.2 In vitro efficacy of contact fungicides against $R$. bataticola

\begin{tabular}{|c|c|c|c|c|c|c|c|c|c|}
\hline \multirow{2}{*}{$\begin{array}{l}\text { Tr. } \\
\text { No. }\end{array}$} & \multirow[t]{2}{*}{ Treatments } & \multicolumn{3}{|c|}{ Colony Dia. *(mm) at ppm } & \multirow{2}{*}{$\begin{array}{l}\text { Av. } \\
(\mathbf{m m})\end{array}$} & \multicolumn{3}{|c|}{$\%$ Inhibition* at ppm } & \multirow{2}{*}{$\begin{array}{c}\text { Av. } \\
\text { Inhibition } \\
(\%)\end{array}$} \\
\hline & & 1500 & 2000 & 2500 & & 1500 & 2000 & 2500 & \\
\hline $\mathbf{T}_{1}$ & Captan 50\% WP & 11.83 & 9.65 & 6.93 & 9.47 & $\begin{array}{c}86.86 \\
(68.74)\end{array}$ & $\begin{array}{c}89.28 \\
(70.89)\end{array}$ & $\begin{array}{c}92.30 \\
(73.89)\end{array}$ & $\begin{array}{c}89.48 \\
(71.07)\end{array}$ \\
\hline $\mathbf{T}_{2}$ & Thiram 75\% WP & 15.19 & 14.17 & 14.12 & 14.49 & $\begin{array}{l}83.13 \\
(65.75)\end{array}$ & $\begin{array}{r}84.26 \\
(66.62)\end{array}$ & $\begin{array}{c}84.31 \\
(66.67)\end{array}$ & $\begin{array}{c}83.90 \\
(66.34)\end{array}$ \\
\hline $\mathbf{T}_{3}$ & Mancozeb 75\% WP & 17.07 & 15.12 & 10.94 & 14.38 & $\begin{array}{c}81.03 \\
(64.18)\end{array}$ & $\begin{array}{c}83.20 \\
(65.80)\end{array}$ & $\begin{array}{c}87.84 \\
(69.60)\end{array}$ & $\begin{array}{c}84.03 \\
(66.44)\end{array}$ \\
\hline $\mathbf{T}_{4}$ & Chlorothalonil 75\% WP & 28.36 & 21.55 & 18.64 & 22.85 & $\begin{array}{c}68.49 \\
(55.85)\end{array}$ & $\begin{array}{l}76.06 \\
(60.70)\end{array}$ & $\begin{array}{l}79.29 \\
(62.93)\end{array}$ & $\begin{array}{c}74.61 \\
(59.74)\end{array}$ \\
\hline $\mathbf{T}_{5}$ & Propineb $70 \%$ WP & 49.86 & 40.97 & 37.92 & 42.92 & $\begin{array}{c}44.60 \\
(41.90)\end{array}$ & $\begin{array}{c}54.47 \\
(47.57)\end{array}$ & $\begin{array}{c}57.86 \\
(49.52)\end{array}$ & $\begin{array}{c}52.31 \\
(46.32)\end{array}$ \\
\hline$T_{6}$ & Copperoxy chloride 50\% WP & 30.80 & 25.90 & 20.55 & 25.75 & $\begin{array}{c}65.77 \\
(54.19)\end{array}$ & $\begin{array}{l}71.22 \\
(57.56)\end{array}$ & $\begin{array}{c}77.17 \\
(61.46)\end{array}$ & $\begin{array}{c}71.39 \\
(57.66)\end{array}$ \\
\hline$\overline{T_{7}}$ & $\begin{array}{l}\text { Carbendazim } 12 \%+ \\
\text { Mancozeb } 63 \% \text { WP }\end{array}$ & 8.19 & 0.00 & 0.00 & 2.73 & $\begin{array}{l}90.90 \\
(72.44)\end{array}$ & $\begin{array}{l}100.00 \\
(90.00)\end{array}$ & $\begin{array}{l}100.00 \\
(90.00)\end{array}$ & $\begin{array}{c}96.97 \\
(79.97)\end{array}$ \\
\hline $\mathbf{T}_{8}$ & $\begin{array}{l}\text { Carbendazim } 25 \%+ \\
\text { Mancozeb } 50 \% \text { WS }\end{array}$ & 19.50 & 16.44 & 12.30 & 16.08 & $\begin{array}{l}78.33 \\
(62.26)\end{array}$ & $\begin{array}{l}81.73 \\
(64.69)\end{array}$ & $\begin{array}{c}86.34 \\
(68.31)\end{array}$ & $\begin{array}{c}82.13 \\
(65.00)\end{array}$ \\
\hline $\mathbf{T}_{9}$ & $\begin{array}{l}\text { Carboxin } 37.5 \% \text { + Thiram } \\
37.5 \% \text { WP }\end{array}$ & 0.00 & 0.00 & 0.00 & 0.00 & $\begin{array}{l}100.00 \\
(90.00)\end{array}$ & $\begin{array}{l}100.00 \\
(90.00)\end{array}$ & $\begin{array}{l}100.00 \\
(90.00)\end{array}$ & $\begin{array}{l}100.00 \\
(90.00)\end{array}$ \\
\hline$T_{10}$ & $\begin{array}{l}\text { Metalaxyl } 8 \%+\text { Mancozeb } \\
64 \% \text { WP }\end{array}$ & 55.62 & 48.94 & 43.75 & 49.43 & $\begin{array}{c}38.20 \\
(38.18)\end{array}$ & $\begin{array}{c}45.63 \\
(42.49)\end{array}$ & $\begin{array}{c}51.39 \\
(45.80)\end{array}$ & $\begin{array}{c}45.07 \\
(42.17)\end{array}$ \\
\hline $\mathrm{T}_{11}$ & Control (untreated) & 90.00 & 90.00 & 90.00 & 90.00 & $\begin{array}{c}0.00 \\
(0.00)\end{array}$ & $\begin{array}{c}0.00 \\
(0.00)\end{array}$ & $\begin{array}{c}0.00 \\
(0.00)\end{array}$ & $\begin{array}{c}0.00 \\
(0.00)\end{array}$ \\
\hline & S.E. \pm & 0.34 & 0.20 & 0.17 & 0.24 & 0.27 & 0.15 & 0.14 & 0.19 \\
\hline & C.D. $(P=0.01)$ & 1.00 & 0.58 & 0.50 & 0.69 & 0.80 & 0.46 & 0.41 & 0.56 \\
\hline & & & & & & Fungicides & Conc. & F X C & \\
\hline & S.E. \pm & & & & & 1.56 & 0.06 & 0.18 & \\
\hline & C.D. $(\mathrm{P}=0.01)$ & & & & & 4.61 & 0.16 & 0.52 & \\
\hline
\end{tabular}

*: Mean of three replications, Dia: Diameter, Av.: Average

Figures in parentheses are arcsine transformed values 

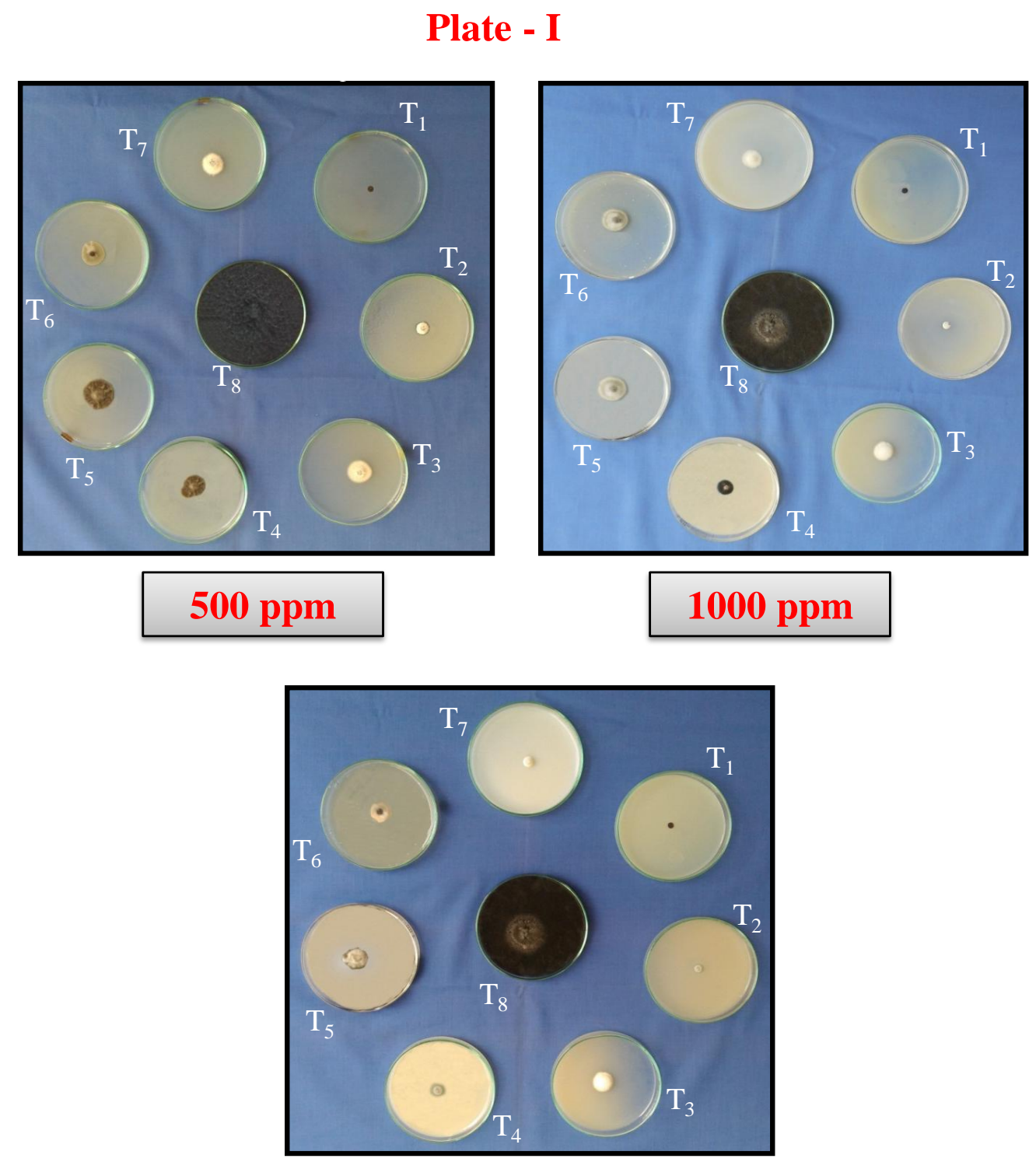

\section{0 ppm}

\section{Tr. No. Treatments}

\begin{tabular}{|l|l|}
\hline $\mathrm{T}_{1}$ & Carbendazim 50\% WP \\
\hline $\mathrm{T}_{2}$ & Thiophanate methyl 70\% WP \\
\hline $\mathrm{T}_{3}$ & Benomyl 50\% WP \\
\hline $\mathrm{T}_{4}$ & Difenconazole 25\% EC \\
\hline
\end{tabular}

\begin{tabular}{|c|l|}
\hline Tr. No. & Treatments \\
\hline $\mathrm{T}_{5}$ & Propaconazole 25\% EC \\
\hline $\mathrm{T}_{6}$ & Tebuconazole 29.9\% EC \\
\hline $\mathrm{T}_{7}$ & Hexaconazole 5\% EC \\
\hline $\mathrm{T}_{8}$ & Control (untreated) \\
\hline
\end{tabular}

In vitro efficacy of systemic fungicides against $R$. bataticola (Rb-4 isolate) 


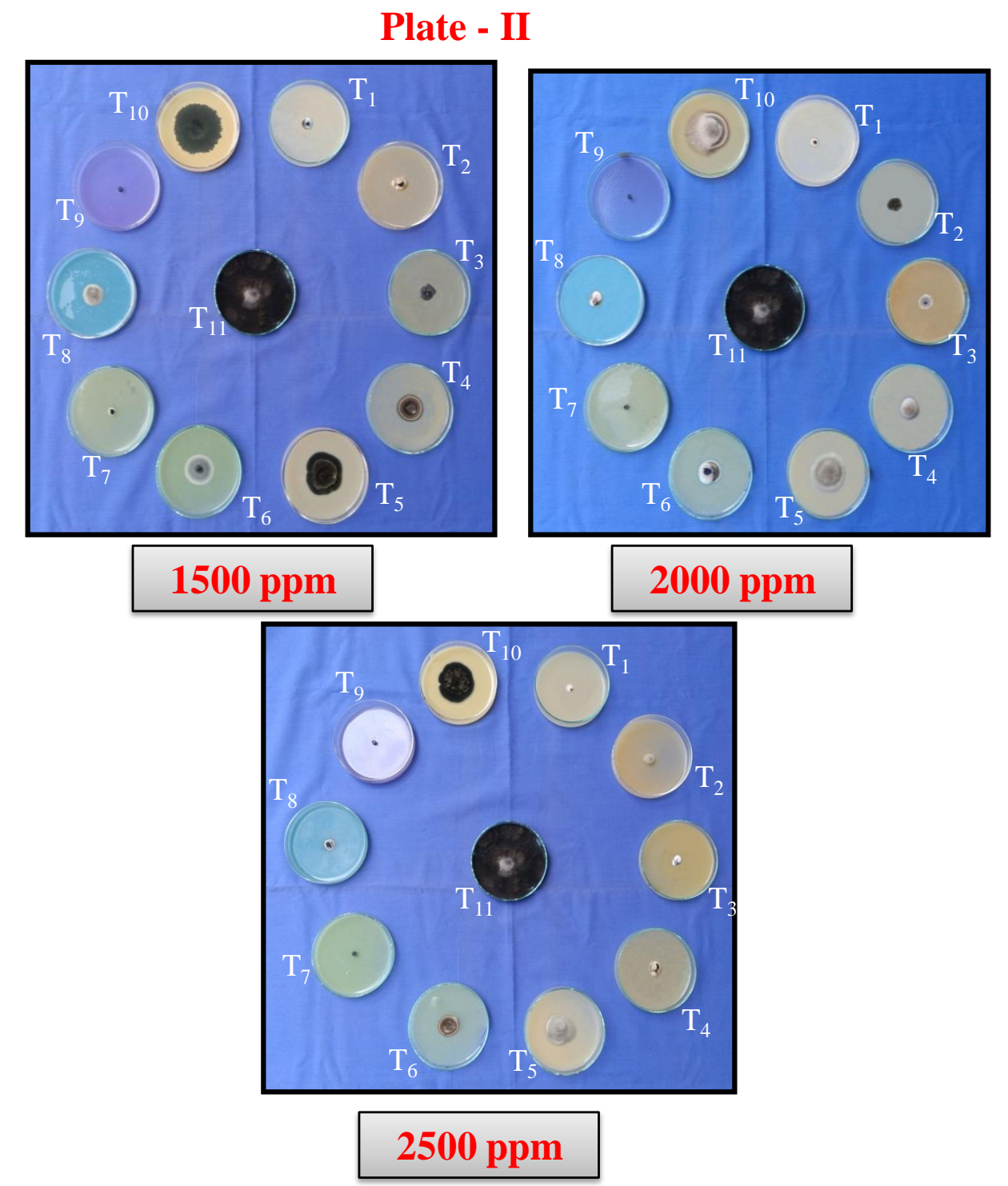

\begin{tabular}{|c|l|}
\hline Tr. No & Treatments \\
\hline $\mathrm{T}_{1}$ & Captan 50\% WP \\
\hline $\mathrm{T}_{2}$ & Thiram75\% WP \\
\hline $\mathrm{T}_{3}$ & Mancozeb 75\% WP \\
\hline $\mathrm{T}_{4}$ & Chlorothalonil 75\% WP \\
\hline $\mathrm{T}_{5}$ & Propineb 70\% WP \\
\hline $\mathrm{T}_{6}$ & Copperoxy chloride 50\% WP \\
\hline
\end{tabular}

\begin{tabular}{|c|l|}
\hline Tr. No. & Treatments \\
\hline $\mathrm{T}_{7}$ & Carbendazim 12\% + Mancozeb 63\% WP \\
\hline $\mathrm{T}_{8}$ & Carbendazim 25\% + Mancozeb 50\% WS \\
\hline $\mathrm{T}_{9}$ & Corboxin 37.5\% + Thiram 37.5\% WP \\
\hline $\mathrm{T}_{10}$ & Metalaxyl 8\% + Mancozeb 64\% WP \\
\hline $\mathrm{T}_{11}$ & Control (untreated) \\
\hline
\end{tabular}

In vitro efficacy of contact and combi-fungicides against $R$. bataticola 
Fig.1 In vitro bio-efficacy of systemic fungicides against $R$. bataticola

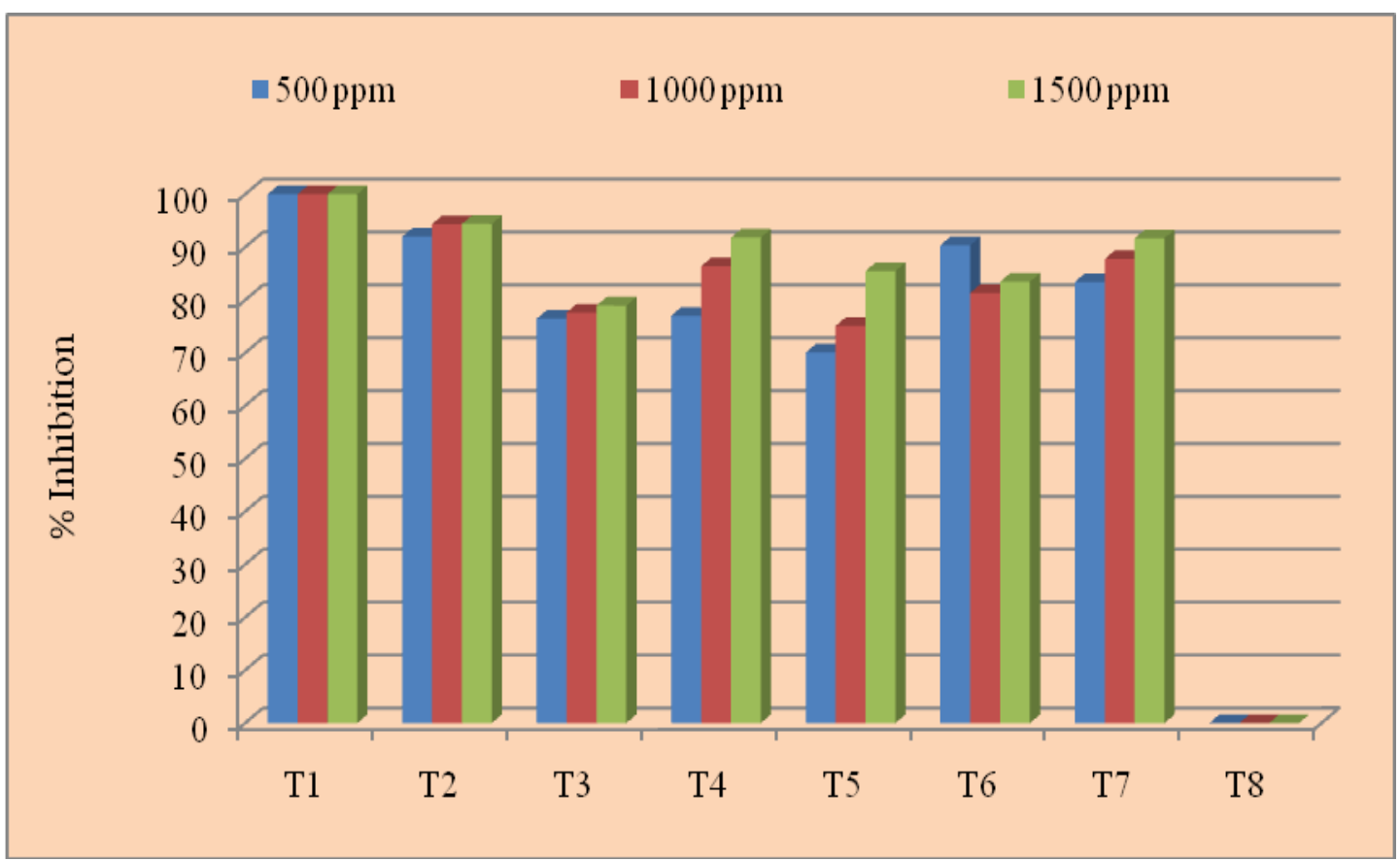

Fig.2 In vitro bio-efficacy of contact and combi-fungicides against $R$. bataticola

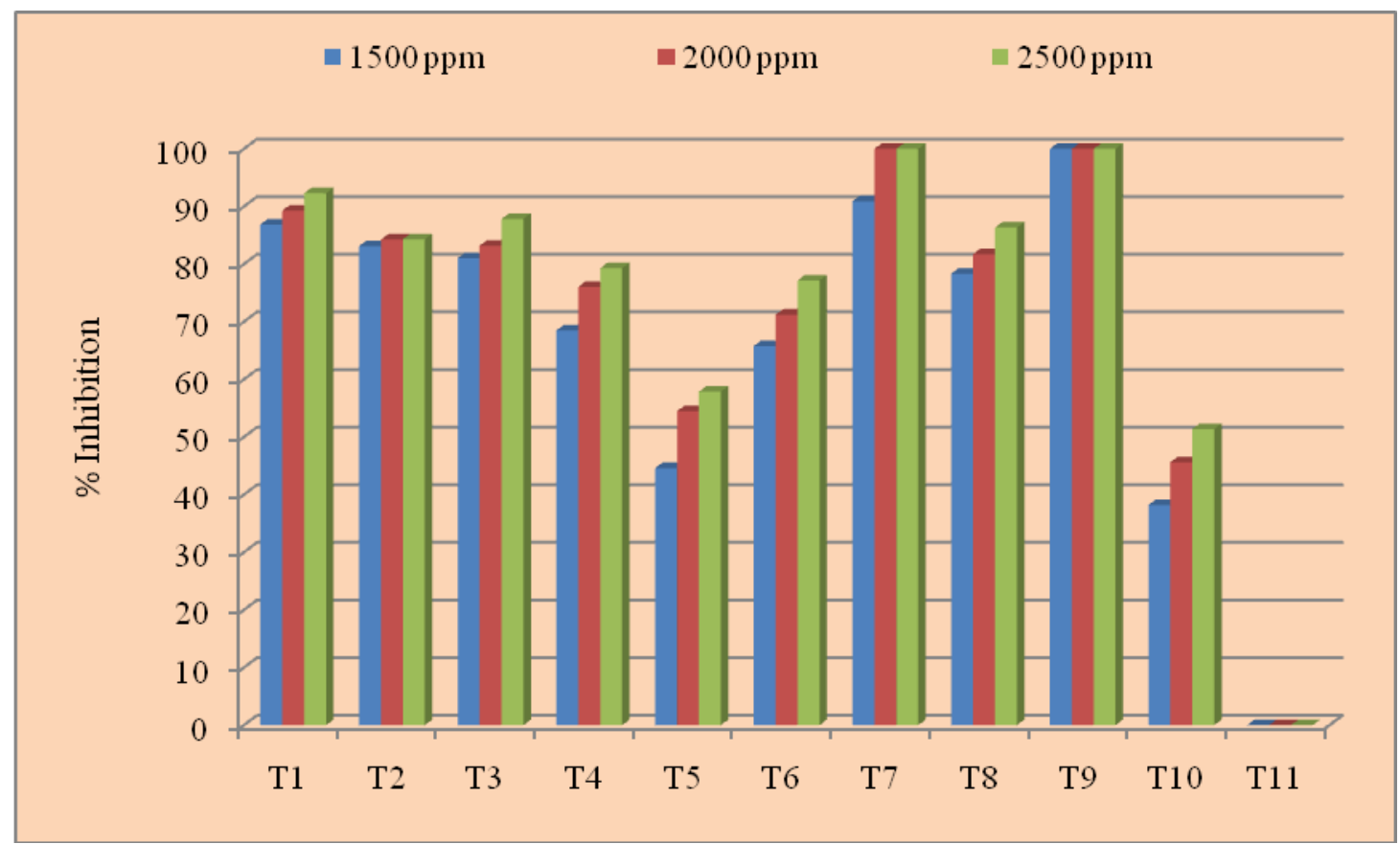

The next best fungicides with significantly least mycelia growth were Captan 50\% WP (11.83, 9.65 and $6.93 \mathrm{~mm}$, respectively), followed by Thiram 75\% WP $(15.19,14.17$ and $14.12 \mathrm{~mm}$, respectively), Mancozeb 75\% WP (17.07, 15.12 and $10.94 \mathrm{~mm}$, respectively) and Carbendazim $25 \%+$ Mancozeb 50\% WS (19.50, 16.44 and $12.30 \mathrm{~mm}$, respectively), each @ 1500, 2000 
and $2500 \mathrm{ppm}$, respectively. Rest of the fungicides tested also resulted with minimum mycelia growth, compare to untreated control (90.00 mm).

Average mycelial growth of the test pathogen ranged from 00.00 to $49.43 \mathrm{~mm}$. However, it was nil with Carboxin $37.5 \%$ + Thiram $37.5 \%$ WP $(0.00 \mathrm{~mm})$, followed by Carbendazim $12 \%$ WP + Mancozeb $63 \%$ WP (2.73 mm), Captan $50 \% \mathrm{WP}(9.47 \mathrm{~mm})$, followed by Mancozeb 75 \% WP (14.38 mm), Thiram $75 \%$ WP (14.49 $\mathrm{mm})$, Carbendazim $25 \%$ + Mancozeb $50 \%$ WS (16.08 mm), Chlorothalonil $75 \%$ WP (22.85 $\mathrm{mm})$, Copperoxy chloride 50 \% WP (25.75 $\mathrm{mm})$, Propineb $70 \% \mathrm{WP}(42.92 \mathrm{~mm})$ and Metalaxyl $8 \%$ + Mancozeb $64 \%$ WP (49.43 $\mathrm{mm})$.

\section{Mycelial growth inhibition}

Results (Table 2, Fig. 2) revealed that all of the contact and combi- fungicides tested (each @ 1500, 2000 and $2500 \mathrm{ppm}$ ) significantly inhibited mycelial growth of $R$. bataticola, over untreated control and it was found to be increased with increase in concentrations of the fungicides tested.

With the contact and combi-fungicides tested, mycelia growth inhibition ranged from 38.20 to 100 per cent, 45.63 to 100 per cent and 51.39 to 100 per cent, respectively @ 1500, 2000 and 2500 ppm. However, Carboxin 37.5\% + Thiram 37.5\% WP (@ 1500, 2000 and 2500 ppm) and Carbendazim $12 \%$ WP + Mancozeb $63 \%$ WP (@2000 and 2500 ppm), resulted with cent per cent $(100 \%)$ mycelial growth inhibition. These were followed by Captan 50\% WP (86.86, 89.28 and $92.30 \%$, respectively), Thiram $75 \%$ WP (83.13, 84.26 and $84.31 \%$, respectively), Mancozeb 70\% WP (81.03, 83.20 and $87.84 \%$, respectively), Carbendazim $25 \%+$ Mancozeb $50 \%$ WS (78.33, 81.73 and 86.34\%, respectively) and Chlorothalonil 75\% WP (68.49, 76.06 and 79.29\%, respectively), @ 1500, 2000 and $2500 \mathrm{ppm}$, respectively). Rest of the fungicides also resulted with significant mycelia growth inhibition, over untreated control. Average mycelial growth inhibition recorded with the test contact and combifungicides ranged from 45.07 to 100 per cent. However, it was cent per cent $(100 \%)$ with Carboxin 37.5 \% + Thiram 37.5 \% WP followed by Carbendazim $12 \% \mathrm{WP}+$ Mancozeb 63 \% WP (96.97\%), Captan $50 \%$ WP (89.48 \%), Mancozeb $75 \%$ WP (84.03\%), Thiram $75 \%$ WP (83.90\%), Carbendazim 25 $\%+$ Mancozeb $50 \%$ WS (82.13\%), Chlorothalonil $75 \%$ WP (74.61\%), Copperoxy chloride $50 \%$ WP (71.39\%), Propineb $70 \%$ WP $(52.31 \%)$ and Metalaxyl $8 \%+$ Mancozeb $64 \%$ WP (45.07\%). Thus, all of the contact and combi-fungicides tested were found fungistatic against $R$. bataticola and significantly inhibited its mycelial growth, over untreated control. However, on the basis of order of merit the contact and combi-fungicides found most effective were Carboxin $37.5 \%+$ Thiram $37.5 \% \mathrm{WP}>$ Carbendazim $12 \% \mathrm{WP}+$ Mancozeb $63 \%$ WP > Captan 50 \% WP > Mancozeb $75 \%$ WP > Thiram $75 \%$ WP > Carbendazim $25 \%$ + Mancozeb $50 \%$ WS.

These results of the present study on in vitro bioefficacy of seven systemic, six contact and four combi-fungicides against $R$. bataticola are in conformity with the earlier findings of several workers, who also reported these fungicides as most effective in inhibiting mycelial growth of $R$. bataticola / $M$. phaseolina, causing dry root rot / charcoal rot of soybean as well as many other crops. Khonde et al., (2008) reported Carbendazim 50 \% WP $(0.1 \%)$, Thiram $75 \%$ WP $(0.3 \%)$, Mancozeb 75 $\%$ WP $(0.25 \%)$, Thiophanate methyl $70 \%$ WP $(0.1 \%)$ and Penconazole $50 \%$ EC $(0.1 \%)$ as most effective in inhibiting mycelial growth of $R$. bataticola, causing root rot of soybean. Magar et al., (2011) reported that Carbendazim 50\% WP@0.1\% and Mancozeb 75 \% WP @ $0.25 \%$ caused 100 per cent mycelial growth inhibition of M. Phaseolina. Khan et al., (2012) reported Mancozeb $75 \% \mathrm{WP}$, Carbendazim 50 $\%$ WP, Benomyl $50 \%$ WP, Copperoxy chloride $50 \%$ WP (each @ $0.2 \%$ ) as effective against $R$. bataticola, causing dry root rot of chickpea. Sangeetha and Jahagirdar (2013) reported 
Carbendazim 50\% WP and Thiophanate methyl $70 \%$ WP caused 100 per cent mycelial growth inhibition of $R$. Bataticola, causing root rot of soybean; whereas, Hexaconazole 5\% EC, Propiconazole $25 \%$ EC, Carbendazim $25 \%+$ Mancozeb $50 \%$ WP, Carboxin $37.5 \%+$ Thiram 37.5\% WP and Mancozeb $75 \%$ WP caused 91.60 to 96.41 per cent mycelial growth inhibition, respectively. Maruti et al., (2017) reported that, Tebuconazole 25.9\% EC (@ 500 ppm), Propiconazole $25 \%$ EC, Hexaconazole 5 $\%$ EC, Difenconazole $25 \%$ EC, Carbendazim $50 \%$ WP and Thiophanate methyl $70 \%$ WP (each@ @00, 1000 and 1500 ppm) resulted with 100 per cent mean mycelial growth inhibition of $R$. bataticola, causing dry root rot of chickpea. The contact fungicides viz., Mancozeb $75 \%$ WP, Thiram $80 \%$ WP, Chlorothalonil $75 \%$ WP and Captan 70 \% WP (each @ 1000, 2000 and $3000 \mathrm{ppm}$ ) and combi-fungicides viz., Carbendazim 12\% + Mancozeb 63\% WP, and Carboxin 37.5\% + Thiram 37.5\% WP (each @ 1000, 2000 and $3000 \mathrm{ppm}$ ) were also effective against $R$. bataticola .

\section{References}

Khan, R. A., Bhat, T. A. and Kumar, K. (2012). Management of chickpea (Cicer arietinum L.) dry root rot caused by Rhizoctonia bataticola (Taub.) Butler. Internat. J. Res. Pharma. Biomedi. Sci. 3 (4): 1539-1548.

Khonde, S. A., Raut, B. T. and Gade, M. R. (2008a). Chemical and biological management of root rot (Rhizoctonia bataticola) of soybean. Ann. Pl. Physiol. 22 (2): 275-277.

Magar, S. V., Kadam, J. J., Rite, S. C., Thaware, D. S. and Potphode, P. D. (2011). In vitro efficacy of fungicides against Macrophomina phaseolina (Tassi.) Goid, causing leaf spot in greengram. Int. J. Pl. Prot. 4 (1): 30-33.

Maruti, Savitha, A. S., Sunkad, G. and Amaresh, Y. S. (2017). In vitro efficacy of fungicides and bio-agents against dry root rot of Pigeonpea caused by Rhizoctonia bataticola (Taub.) Butler.

Muthuswamy, S. and Mariappan, V. (1991). Disintegration of sclerotia of Macrophomina phaseolina, (Soybean isolate) by oil cake extract. Indian Phytopath. 44: 271-273.

Nene, Y. L. and Thapliyal, R. N. (1993). Evaluation of fungicides. In: Fungicides in Plant Disease Control ( $3^{\text {rd }}$ edn.), Oxford, IBH Pub. Co. Pvt. Ltd., New Delhi. Pp. 525-542.

Sangeetha, T. V. and Jahagirdar, S. (2013a). Survey for the assessment of disease incidence of root rot/wilt of soybean in northern Karnataka. Karnataka J. Agric. Sci. 26 (4): 563-564.

Sonka, S. T., Bender, K. L. and Fisher, D. K. (2004). Soybeans: Improvement, production and uses. ASA, CSSA, and SSSA. Madison, WI, USA. 3: 919-948.

Vincent, J. M. (1927). Distortion of fungal hyphae in the presence of certain inhibitors, Nature. Pp. 159-180.

\section{How to cite this article:}

Agale, R.C., A.P. Suryawanshi, R.R. Rathod and Apet, K.T. 2018. Bioefficacy of Various Fungicides against Rhizoctonia bataticola, Causing Dry Root Rot of Soybean. Int.J.Curr.Microbiol.App.Sci. 7(10): 1856-1864. doi: https://doi.org/10.20546/ijcmas.2018.710.213 